\title{
Review of: "Evaluation of automated specialty palliative care in the intensive care unit: $A$ retrospective cohort study"
}

\author{
Alejandra Fernández Trujillo
}

Potential competing interests: The author(s) declared that no potential competing interests exist.

The possibility of a palliative consultor in the ICU is not data that can be extrapolated to other settings. The reasons for consultation do not correspond closely to those present in the cited recommendations, which indicates that despite these recommendations there is no protocol (as indicated in the study). It is possible that patients were left out with the recommended criteria, but there was a high proportion of patients who met them and who were not consulted.

The exact role of the palliativist in the ICU is not well defined, which can be a limitation when it comes to consultation.

One limitation is that it is a retrospective study. It could be interesting a prospective study and possibly determinate other triggers for palliative calling.

The fact that the decision ultimately depends on subjectivity (despite the publication of reasons for consultation) is one more reason to individualize this process, since, as we have seen, with rigid criteria there are patients who may be left out of this care .

However, I believe that in a comprehensive ICU model, intensivists should have the necessary training to deal with end-of-life situations and have palliative care in cases where the care process continues outside the unit. Furthermore, in this context, other professionals who can provide psychological or spiritual support to the patient or their families would be as important as the palliative doctor.

In JP Bishop's article "Efficient, compassionate, and fractured: contemporary care in the ICU", the interviewed doctors considered that patients who were nearing the end of life were the object of palliativists to make a more efficient medicine, since they they did not have time for compassionate care due to having to spend time with those who would survive. In this case, I believe that intensivists need training in this all the more so, not only because patients who are going to die should be cared for by them but because those who will survive also have the right to compassionate (as well as efficient) care. 